\title{
Influence of Solution and Spinning Parameters on Nanofiber Mat Creation of Poly(ethylene oxide) by Needleless Electrospinning
}

\author{
Timo GROTHE, Johannes BRIKMANN, Hubert MEISSNER, Andrea EHRMANN* \\ Bielefeld University of Applied Sciences, Faculty of Engineering and Mathematics, 33619 Bielefeld, Germany \\ crossref http://dx.doi.org/10.5755/j01.ms.23.4.17169
}

Received 30 November 2016; accepted 14 April 2017

\begin{abstract}
Electrospinning allows producing fine polymer fibers with diameters in the range of several hundred nanometers up to some micrometers. While a large amount of polymers necessitates spinning either from melt or from solutions which are hazardous to health and environment, biopolymers and some other materials are water-soluble and thus can be spun from pure water or similar uncritical (i.e. non-toxic, non-hazardous) solutions. Electrospinning from aqueous solutions, however, is from the technological point of view often more complicated than using other solvents, since water evaporates slower and requires careful designing of the spinning process parameters. This article gives an overview of the influence of spinning and material parameters on nanomats produced from poly(ethylene oxide) (PEO, also known as poly(ethylene glycol), PEG), depicting which parameters are suitable for needleless electrospinning, opposite to parameters found in the literature for nanospinning with a needle.
\end{abstract}

Keywords: nanospinning, electrospinning, PEO, PEG.

\section{INTRODUCTION}

Fibers spun by traditional methods, such as melt spinning or spinning from solution, usually result in fibers with diameters in the range of some micron or smaller. Ultra-fine fibers (finer than 0.3 dtex, i.e. diameters in the range of some hundred nanometers) were produced as stable fibers with random length [1] or continuous filament [2] several decades ago.

Electrospinning, also called nanospinning, is another method which can be used to produce either continuous fibers or fiber mats composed of fibers with consistent diameters [3-7]. This technology offers a relatively easy possibility to create ultrathin fibers from a broad range of materials, i.e. polymers, composites and even ceramics. Such nanofibers are especially versatile in applications which necessitate large surfaces areas, e.g. for promotion of cell growth in biomedical applications, as a catalyzer [8], novel filter materials [9-11] or medical wound dressing [12-14].

Electrospinning can create fibers using a needle through which a polymer is pressed which is afterwards drawn to a substrate by an electrical field between needle and substrate. In needleless electrospinning, an electrical field is applied between two wires, one of which is coated by a polymer solution (or melt). The electrical field forces the coating to be drawn to the counter electrode which is shielded by a movable substrate, building so-called Taylor cones, i.e. cone-shaped jets of fibers [15]. The fibers are stretched and thinned on their path between the electrodes.

While typical organic solvents evaporate quite fast, spinning from aqueous solutions results in much slower evaporation processes, possibly leading to wet areas on the substrate and thus undesired agglutinating of fibers. Nevertheless, "green" electrospinning - which abstains

\footnotetext{
* Corresponding author. Tel.: +49521 10670254; fax: +49 5211067190 .

E-mail address: andrea.ehrmann@fh-bielefeld.de (A. Ehrmann)
}

from hazardous solvents - is of great interest in recent research. These fibers can either be stabilized in a second step to enhance their water-stability [16-19], or the watersoluble polymer serves as a matrix in which a not watersoluble partner is embedded which is co-spun and remains when the water-soluble parts of the fibers are dissolved from the fiber mats [20-22].

An interesting water-soluble polymer is polyethylene glycol (PEG or PEO) which is used in a variety of applications, from medicine and pharmaceutics to green wood stabilization to skin creams and toothpaste. PEG is often used as coating or supplement for fibers created from other materials $[23,24]$ or spun as composite fiber in combination with other materials, such as cellulose, collagen or PVDF [25-27]. However, reports about spinning of pure PEO fibers are scarce [28, 29].

An interesting article of Deitzel et al. describes the influence of different concentrations and process parameters on needle electrospun nanofibers from PEG in detail [30]. Since these parameters differ strongly from those useful in needleless electrospinning of PEO nanofiber mats, our article gives an overview of the effects of diverse spinning parameters for needleless nanospinning of PEO.

\section{EXPERIMENTAL}

Polymer solutions were prepared from PEO with a molecular weight of 600000 daltons purchased from S3 Chemicals. The PEO was stirred with distilled water for 2 hours when all clusters are dissolved. Solutions with PEO contents between $2 \%$ and $8 \%$ were prepared.

For electrospinning, the needleless nanospinning machine "Nanospider Lab" (Elmarco, Czech Republic) was used. Spinning parameters were in the ranges given in Table 1 and are defined exactly in the description of the respective results. Tests were performed with $5-10 \mathrm{ml}$ of spinning solution. 
Table 1. Parameter ranges for the electrospinning process

\begin{tabular}{|c|c|c|}
\hline Parameter & $\min$ & $\max$ \\
\hline Voltage, $\mathrm{kV}$ & 25 & 80 \\
\hline Current, $\mathrm{mA}$ & 0.006 & 0.25 \\
\hline Nozzle diameter, $\mathrm{mm}$ & 0.6 & 0.9 \\
\hline Carriage speed, $\mathrm{mm} / \mathrm{s}$ & 100 & 300 \\
\hline Substrate speed, $\mathrm{mm} / \mathrm{min}$ & 5 & 10 \\
\hline Ground-substrate distance, $\mathrm{mm}$ & 25 & 25 \\
\hline Electrode-substrate distance, $\mathrm{mm}$ & 140 & 230 \\
\hline Rel. humidity in chamber, $\%$ & 29 & 60 \\
\hline Spinning duration, min & 4 & 40 \\
\hline
\end{tabular}

For optical evaluation of the nanomats, a confocal laser scanning microscope (CLSM) VK 9000 by Keyence was used. The images shown in this article have a nominal magnification of $2000 \mathrm{x}$.

The mass per unit area was measured by cutting circles of area $1 \mathrm{dm}^{2}$ using a circular cutter and weighing the fiber mats on an analytical balance.

\section{RESULTS AND DISCUSSION}

The electrical forces dragging the polymer solution from one electrode wire to the other - or the substrate in front of the ground electrode, respectively - are defined by the voltages between both electrodes and the spinning currents. The currents vary with the voltage as well as with the electrode-electrode distance, as depicted in Fig. 1.

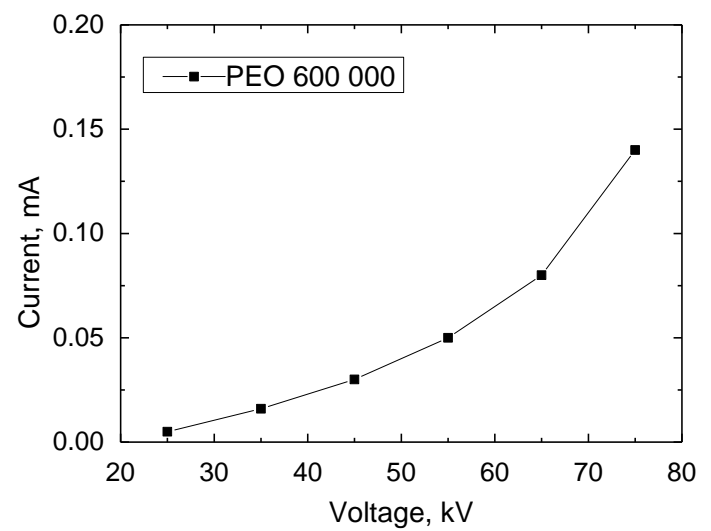

a

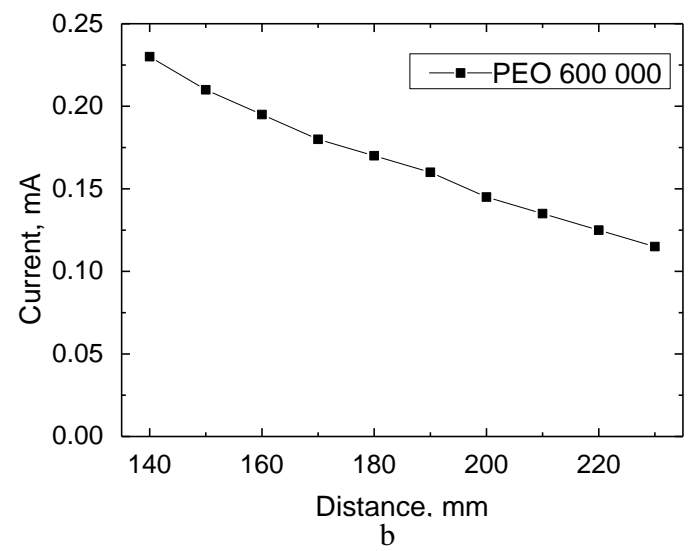

Fig. 1. a-spinning current as a function of voltage, measured for $8 \%$ PEG 600000 in aqueous solution at a constant substrate-electrode distance of $210 \mathrm{~mm}$; b-spinning current as a function of electrode-distance for a constant voltage of $75 \mathrm{kV}$
While the current is approximately linearly correlated with the electrode distance (Fig. 1 a), it increases stronger than linearly with the voltage (Fig. $1 \mathrm{~b}$ ). The dependence of the current on the voltage differs from the finding described in [30] and [31] for needle electrospinning which is attributed to a second-order transition.

The flow rates of the polymer solution have been measured for different PEO concentrations (Fig. 2) and different voltages (Fig. 3), respectively. For a given voltage, the flow rate drastically decreasing with increasing amount of PEO in the solution.

Careful observation of the spinning process shows that on the one hand, "stronger" Taylor cones containing more material are formed for lower fractions of PEO. On the other hand, the spinning nozzle tends to being blocked faster and faster for higher concentrations. This finding is independent of the nozzle diameter - all nozzles between $0.6 \mathrm{~mm}$ and $0.9 \mathrm{~mm}$ diameter which belong to the standard equipment of the Nanospider were tested and found to get choked after short times (<1 min.) for higher concentrations. This results in irregularities in the spinning process, with varying numbers of Taylor cones and thus varying mass distributions on the substrate.

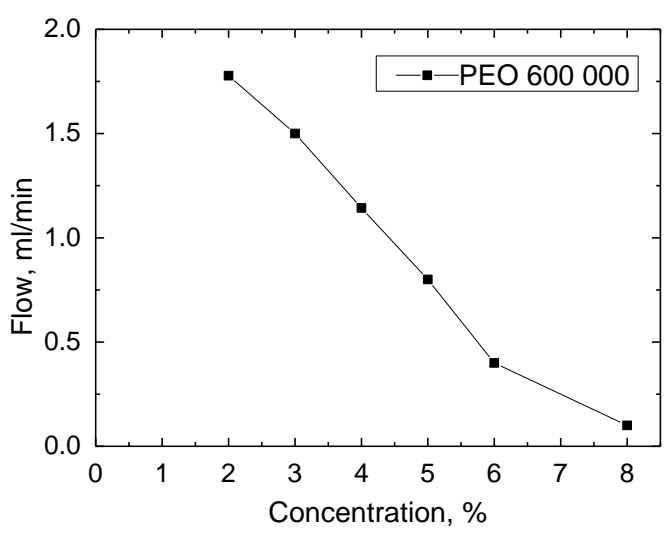

Fig. 2. PEO concentration dependent flow rate, measured for PEO 600000 spun with $65 \mathrm{kV}$ and $0.08 \mathrm{~mA}$, using a nozzle of diameter $0.8 \mathrm{~mm}$, a carriage speed of $250 \mathrm{~mm} / \mathrm{s}$ and a substrate-electrode distance of $210 \mathrm{~mm}$.

On the other hand, higher voltages can be used to enhance the flow rate (Fig. 3).

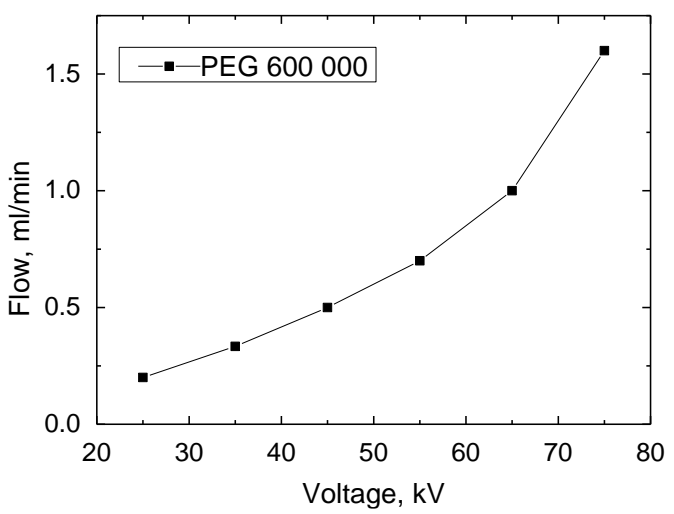

Fig. 3. Voltage dependent flow rate, measured for PEO 600000 spun with $5 \%$ PEO concentration, using a nozzle of diameter $0.8 \mathrm{~mm}$, a carriage speed of $250 \mathrm{~mm} / \mathrm{s}$ and a substrate-electrode distance of $210 \mathrm{~mm}$ 
While the influence of the voltage on the fiber formation will be described later, it should be mentioned here that for the lowest possible voltages which enable "flying" of the polymer material, the Taylor cones are deformed to nearly straight lines. These "jets" seem to be built at irregular, random positions along the wire, resulting in an irregular pattern of substrate areas with a dense fiber distribution, alternating with nearly empty areas. Thus these lowest voltages where fibers start "flying" should be avoided; instead care should be taken during the experiment that proper Taylor cones are formed to enable a regular fiber distribution on the substrate.
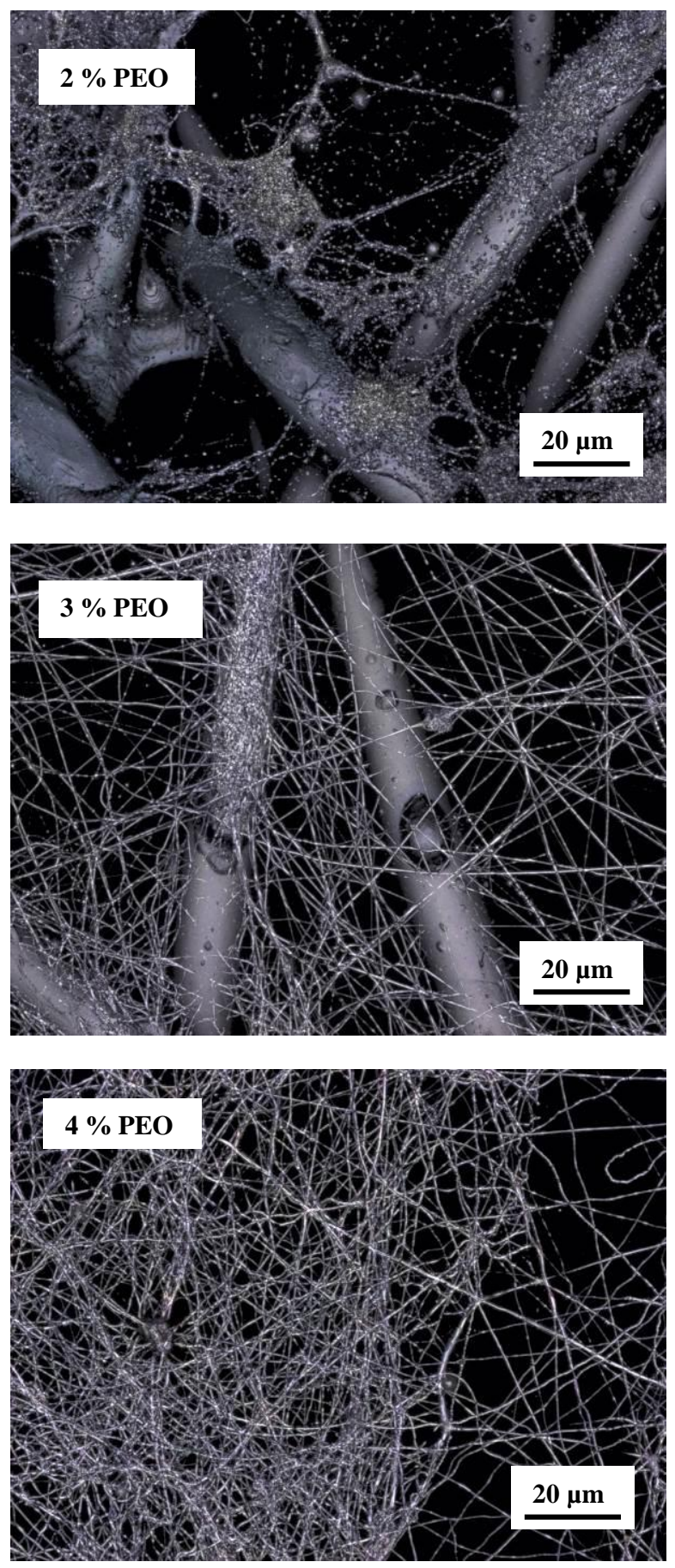

Fig. 4. Microscopic images of nanospun fiber mats, produced from different concentrations of PEO 600000 (full caption in the next column)


Fig. 4. Microscopic images of nanospun fiber mats, produced from different concentrations of PEO 600000 with $65 \mathrm{kV}$ and $0.08 \mathrm{~mA}$, using a nozzle of diameter $0.8 \mathrm{~mm}$, a carriage speed of $250 \mathrm{~mm} / \mathrm{s}$, a substrate-electrode distance of $210 \mathrm{~mm}$ and a relative humidity of $35 \%$

It is known [30] that the amount of fibers strongly depends on the PEO concentration in the aqueous solution. The concentrations found there to be ideal $(\sim 10 \%$ PEO content) are higher than those which can be spun in needleless electrospinning. Thus Fig. 4 shows a series of fiber mats gained by spinning aqueous solutions with different PEO contents. As the images clearly show, for a concentration of $2 \%$ the spinning process mostly produces drops, while fiber formation starts at a concentration of $3 \%$. For $5 \%$ and $6 \%$, the fiber mats become denser. 
Finally, for $8 \%$ PEO the fibers are visibly thicker than for lower concentrations, combined with a smaller amount of fibers due to the reduced flow rate (Fig. 2). The droplet formation for low PEO concentrations can be attributed to the fibers still being wet when reaching the substrate, where they break into droplets due to their surface tension [30].

However, the fiber mat density and appearance is not only influenced by the PEO concentration but also by the voltage. As depicted in Fig. 3, it can be expected that with higher voltages, more fibers are placed on the substrate. Fig. 5 depicts a series of microscopic images of fiber mats produced by different voltages.
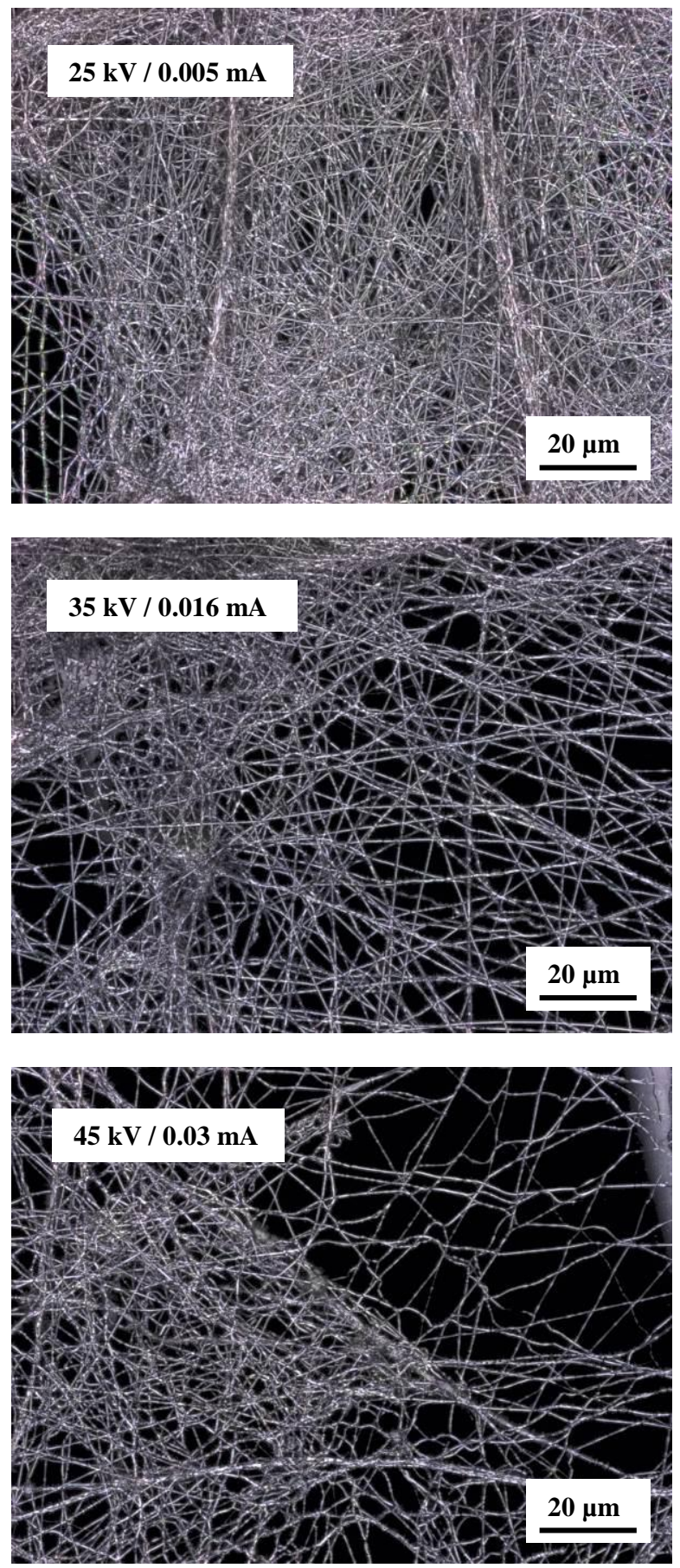

Fig. 5. Microscopic images of nanospun fiber mats, produced with different voltages to spin $5 \%$ PEO 600000 (full caption in the next column)


Fig. 5. Microscopic images of nanospun fiber mats, produced with different voltages to spin 5\% PEO 600000 in aqueous solution, using a nozzle of diameter $0.8 \mathrm{~mm}$, a carriage speed of $250 \mathrm{~mm} / \mathrm{s}$, a substrate-electrode distance of $210 \mathrm{~mm}$ and a relative humidity of $35 \%$

For $25 \mathrm{kV}$, an area was chosen were a high amount of fibers could be found. Here, two thick "vertical" fiber agglomerates are visible which is typical for relatively low voltages and has always been found under these conditions. For $35 \mathrm{kV}$ and $45 \mathrm{kV}$, large areas with only few fibers are depicted, while for higher voltages these areas are reduced and the mats become more regular. Nevertheless, in all pictures it is visible that there are distinct irregularities.

This means that if such a nanofiber mat is to be used as a filter material, it should be produced with reduced 
substrate speed to increase regularity and avoid areas with small amounts of fibers and thus large open pores.

Fig. 6 depicts the fiber diameters, measured $10 \mathrm{x}$ per sample, for different parameters. In [30] it was shown that for needle electrospinning, the fiber diameters increase with the PEO concentration. This is consistent with the finding that the nanofiber diameter is proportional to the viscosity and can thus be modified significantly by sonication [32]. Fig. 6 a reveals the same behavior, while due to the large standard deviations it is not possible to state whether the relation between fiber diameters and PEO concentration is linear, double-logarithmic [30] or different. It should be mentioned, however, that a large number of the "thick" fibers turn out to be composed of two or more single fibers on closer inspection.

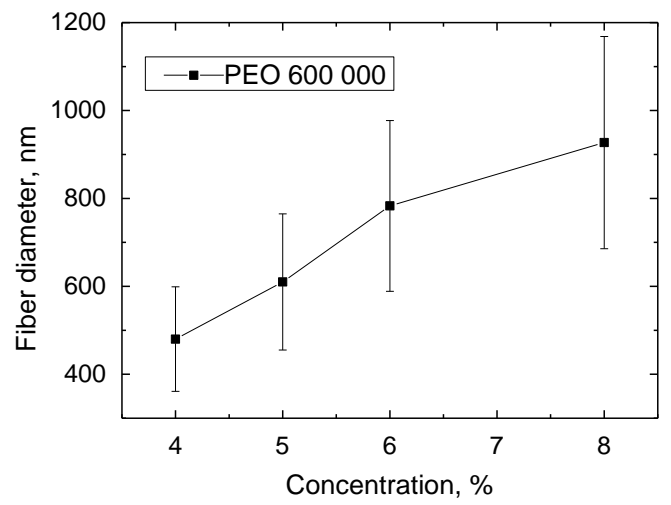

a

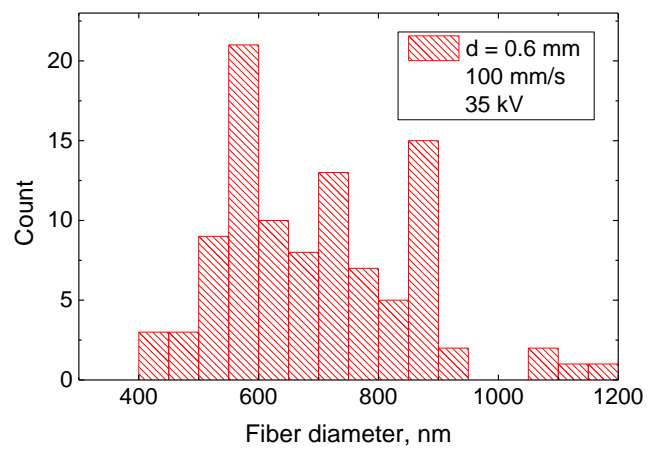

b

Fig. 6. Fiber diameters revealed with a-different PEO concentrations spun with $65 \mathrm{kV}$ and $0.08 \mathrm{~mA}$, using a nozzle of diameter $0.9 \mathrm{~mm}$, a carriage speed of $250 \mathrm{~mm} / \mathrm{s}$ and a substrate-electrode distance of $210 \mathrm{~mm}$; b-fiber diameters distribution, revealed for one of the average measurement points for 100 measured fibers

Fig. $6 \mathrm{~b}$ shows exemplarily the fiber diameter distribution for a nozzle diameter of $0.6 \mathrm{~mm}$, a carriage speed of $100 \mathrm{~mm} / \mathrm{s}$ and a high voltage of $35 \mathrm{kV}$, calculated for 100 measured values of randomly chosen fibers. While the mathematical analysis of the nanofiber diameter distribution was shown by Malasauskiene et al. to be describable by a compound normal distribution [33-35], the values measured here without automation are not sufficient to prove this statement for the nanofiber mats depicted. However, the histogram shows three maxima which may be related to the above mentioned fact that fibers sometimes agglomerate and are thus often described with higher diameters than single fibers.

In Fig. 7, the fiber diameters are depicted in dependence of the spinning voltage for two different nozzle diameters and two different carriage speeds. None of these parameters seems to have a significant influence on the fiber diameter. A slight decrease of the average diameter with increasing voltage is possible but cannot be verified due to the broad distribution of measured values. In any case, these parameters cannot be used to adjust the average fiber diameter.

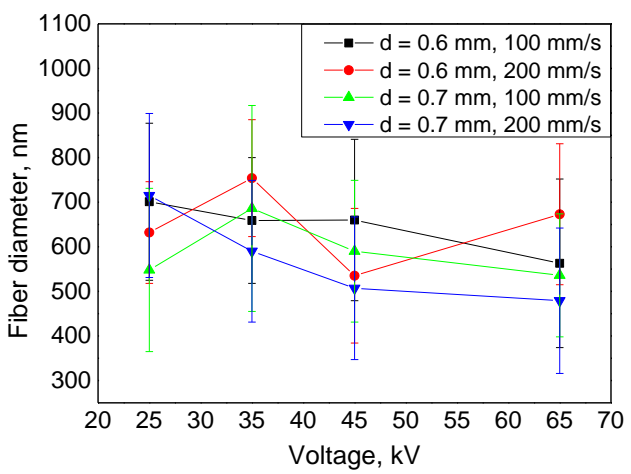

Fig. 7. Fiber diameters revealed for different voltages, nozzle diameters d, carriage speeds of $100 \mathrm{~mm} / \mathrm{s}$ or $200 \mathrm{~mm} / \mathrm{s}$ and a substrate-electrode distance of $210 \mathrm{~mm}$

Fig. 8 depicts the areal weight in dependence of the PEO concentration and the voltage.
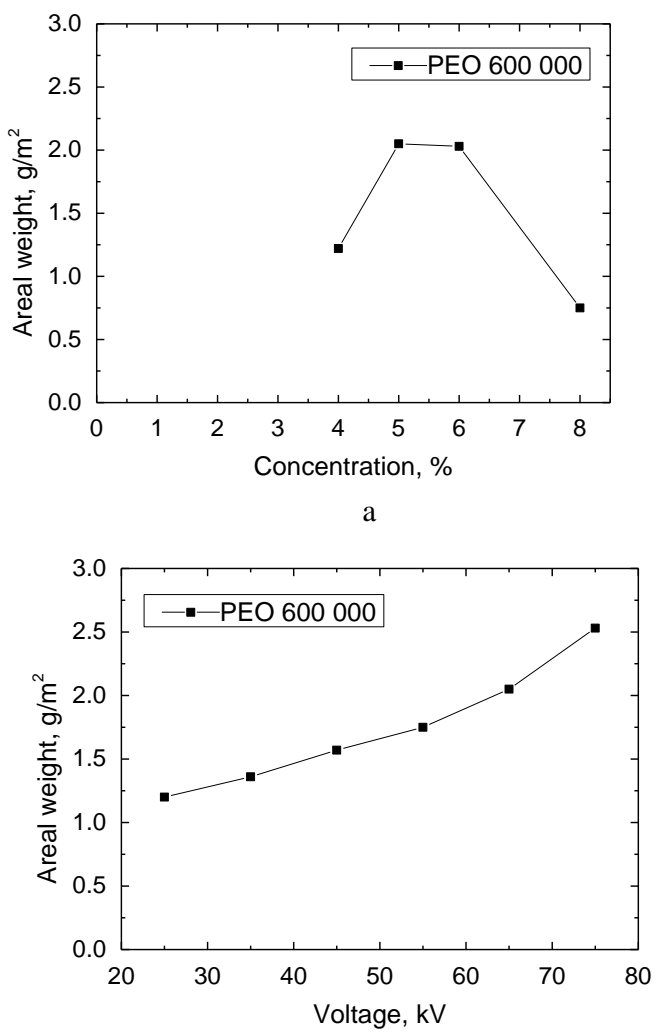

b

Fig. 8. Areal weights measured in dependence of with: a - different PEO concentrations spun with $65 \mathrm{kV}$ and $0.08 \mathrm{~mA}$; or $\mathrm{b}$-different voltages, using a nozzle of diameter $0.8 \mathrm{~mm}$, a carriage speed of $250 \mathrm{~mm} / \mathrm{s}$ and a substrate-electrode distance of $210 \mathrm{~mm}$ 
While the areal weight as a function of the voltage is quite similar to the course of the voltage-dependent flow rate (Fig. 3), the concentration dependence shows a maximum, opposite to the decreasing slope of the concentration-dependent flow (Fig. 2), but in accordance with Fig. 4. This can be explained as follows: For low concentrations, the flow rate is high, but nearly no fibers are created. For high concentrations, the flow rate is low, but the material is in most cases adhered on the substrate where it builds a strong fiber mat. An optimum for these two counteracting effects can apparently be found in the concentration range of 5-6\% PEO.

During peeling the nanofiber mat off the substrate, it was realized that the mats prepared from higher PEO solutions were much more stable; for $8 \%$ concentration the mat could be removed in one part without any problems, while for $6 \%$ care had to be taken not to break it, and the mats prepared from $5 \%$ and $4 \%$ PEO were destroyed during this process. This underlines the importance of choosing the ideal concentration carefully.

Finally, Fig. 9 shows a series of microscopic images taken on nanofiber mats produced at varying relative humidity. For electrospinning, the relative air moisture is said to be crucial; the process is generally claimed to work only at a relative humidity below $40 \%$ which can be hard to reach in not properly air-conditioned laboratories. Other groups have already found significant influence of the relative humidity on the stretching process and thus on the final fiber diameter [36].

Thus we have performed experiments with PEO concentrations of $3 \%$ and $5 \%$ whilst air-conditioning the spinning chamber from $40 \%$ to $60 \%$ relative humidity.

As shown in Fig. 9, the solution with $3 \%$ PEO needs a relative humidity of $\sim 46 \%$ to form fibers, while for a concentration of $5 \%$, fiber formation already starts at $\sim 52 \%$ relative humidity and increases with lower relative humidity. This means that, depending on the PEO concentration, the relative humidity should maximally be in the range of $45-50 \%$, but it not strictly limited to $40 \%$.

The main impact of too high humidity on fiber formation is based on the problem that the water from the aqueous solution has to be evaporated before fibers can be formed and keep their shape while being deposited on the substrate. If the fibers cannot dry fast enough, they onglomerate on the substrate and form droplets instead of the desired fibers.

Besides, the flow rates and fiber diameters do not significantly differ due to relative humidity, but are constant within the measurement accuracy.

\section{CONCLUSION AND OUTLOOK}

To conclude, we have shown that PEO nanofiber formation in needleless electrospinning is influenced by several parameters, with the PEO concentration in the aqueous solution being most crucial, while other parameters such as voltage, electrode-substrate distance, carriage speed etc. only influence the fiber mat density. For PEO 600 000, concentrations between 5 and $6 \%$ were found to be ideal for the formation of a dense, regular nanofiber mat, unlike needle-electrospinning for which significant percentages of fibers were only found at concentrations of $\min .10 \%$.
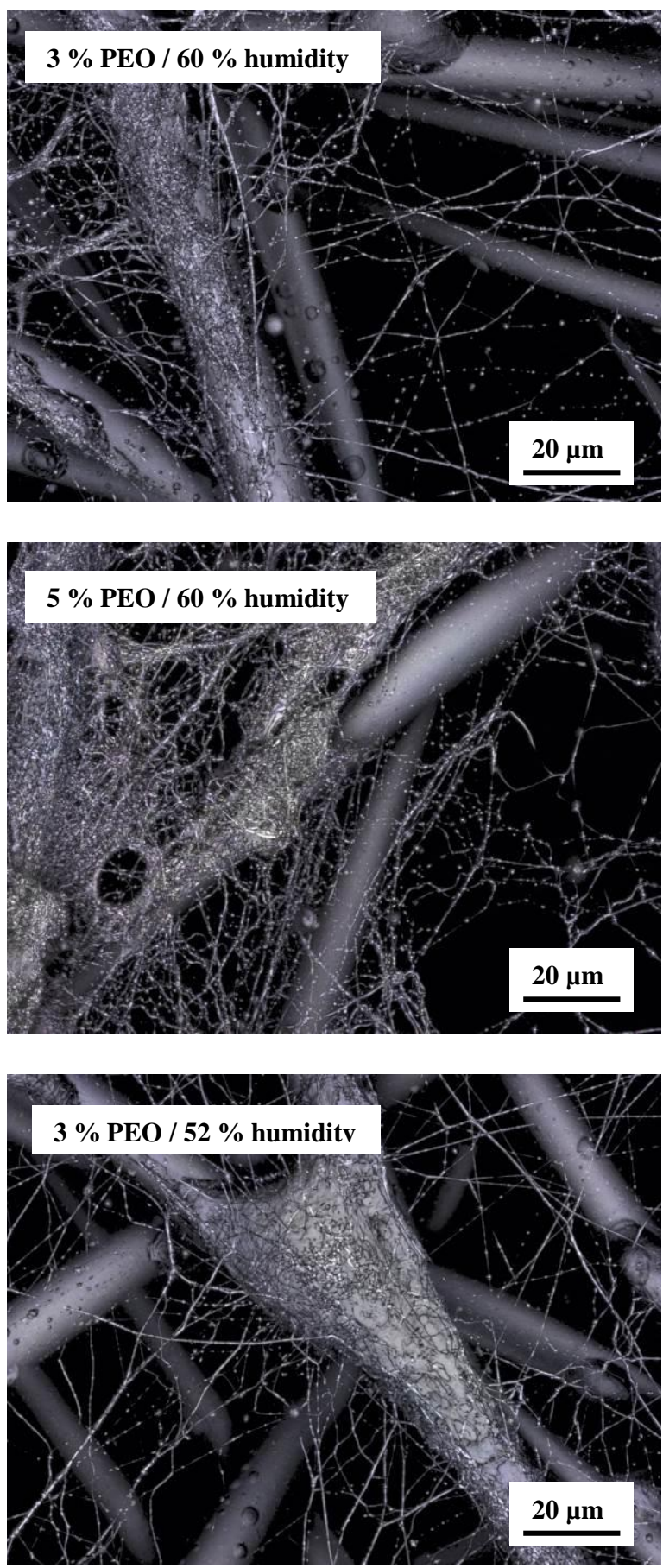

Fig. 9. Microscopic images of nanospun fiber mats, produced with different relative humidity from $3 \%$ and $5 \%$ PEO 600000 (continuation of figure and full caption on next page)

Additionally, fiber diameters reached by needleless electrospinning were generally higher than those obtained by needle electrospinning.

Future experiments will concentrate on the influence of the molecular weight and different possibilities to blend PEO with other materials, especially for applications in medicine and energy textiles. 

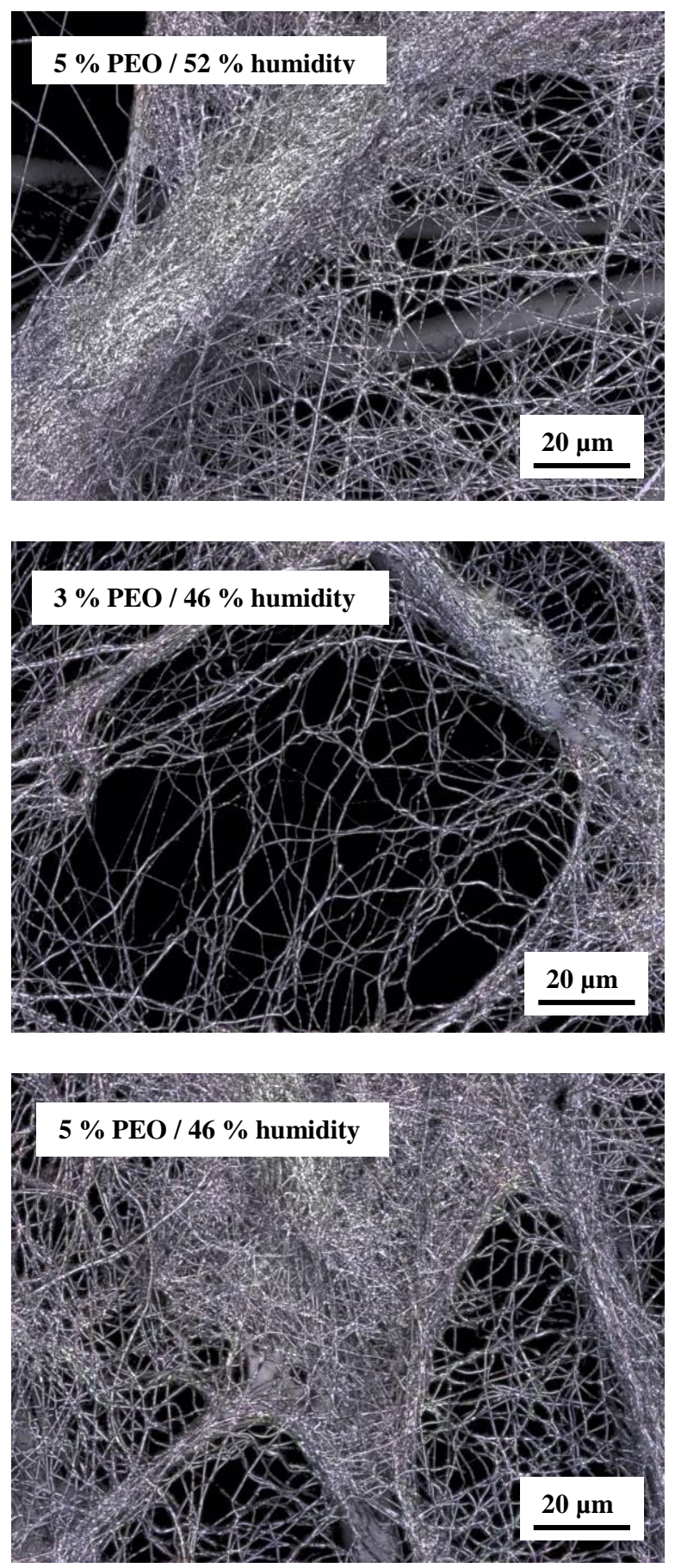

Fig. 9. Microscopic images of nanospun fiber mats, produced with different relative humidity from $3 \%$ and $5 \%$ PEO 600000 , respectively, using a nozzle of diameter $0.8 \mathrm{~mm}$, a voltage of $65 \mathrm{kV}$, a carriage speed of $250 \mathrm{~mm} / \mathrm{s}$ and a substrate-electrode distance of $210 \mathrm{~mm}$.

\section{Acknowledgment}

The authors acknowledge gratefully the program $\mathrm{FH}$ Basis of the German federal country North RhineWestphalia for funding the "Nanospider Lab".

\section{REFERENCES}

1. Nakajima, T., Kajiwara, K., McIntyre, J.E. Advanced Fiber Spinning Technology, Woodhead Publishing 1994. https://doi.org/10.1533/9781845693213
2. Kanigel, R. Faux real: Genuine Leather and 200 Years of Inspired Fakes, Joseph Henry Press 2007.

3. Subbiah, T., Bhat, G.S., Tock, R.W., Parameswaran, S., Ramkumar, S.S. Electrospinning of Nanofibers Journal of Applied Polymer Science 96 2005: pp. 557-569. https://doi.org/10.1002/app.21481

4. Greiner, A., Wendorff, J.H. Electrospinning: A Fascinating Method for the Preparation of Ultrathin Fibers Angewandte Chemie International Edition 46 2007: pp. 5670-5703.

5. Li, D., Xia, Y. Electrospinning of Nanofibers: Reinventing the Wheel? Advanced Materials 16 2004: pp. $1151-1170$. https://doi.org/10.1002/adma.200400719

6. Teo, W.E., Inai, R., Ramakrishna, S. Technological Advances in Electrospinning of Nanofibers Science and Technology of Advanced Materials 12 2011: no. 013002.

7. Agarwal, S., Greiner, A., Wendorff, J.H. Electrospinning of Manmade and Biopolymer Nanofibers - Progress in Techniques, Materials, and Applications Progress in Polymer Science 38 2013: pp. 963-991. https://doi.org/10.1016/j.progpolymsci.2013.02.001

8. Wang, X., Kim, Y.G., Drew, C., Ku, B.C., Kumar, J., Samuelson, L.A. Electrostatic Assembly of Conjugated Polymer Thin Layers on Electrospun Nanofibrous Membranes for Biosensors Nano Letters 4 2004: pp. 331-334.

9. Lackowski, M., Krupa, A., Jaworek, A. Nonwoven Filtration Mat Production by Electrospinning Method Journal of Physics: Conference Series 301 2011: no. 012013.

10. Filatov, Y. Electrospinning of Micro- and Nanofibers: Fundamentals and Applications in Separation and Filtration Processes, Begell House Inc. 2007.

11. Lemma, S.M., Esposito, A., Mason, M., Brusetti, L., Cesco, S., Scampicchio, M. Removal of Bacteria and Yeast in Water and Beer by Nylon Nanofibrous Membranes Journal of Food Engineering 137 2015: pp. 1-6.

12. Huang, Z.M., He, C.K., Yang, A., Zhang, Y., Han, X.J., Yin, J., Wu, Q. Encapsulating Drugs in Biodegradable Ultrafine Fibers Through Co-axial Electrospinning Journal of Biomedical Materials Research Part A $\quad 77$ 2006: pp. 169-179.

13. Ashammakhi, N., Ndreu, A., Yang, Y., Ylikauppila, H., Nikkola, L. Nanofiber-based Scaffolds for Tissue Engineering European Journal of Plastic Surgery 35 2012: pp. $135-149$. https://doi.org/10.1007/s00238-008-0217-3

14. Xie, J., MacEwan, M.R., Schwartz, A.G., Xia, Y. Electrospun Nanofibers for Neural Tissue Engineering Nanoscale 2 2010: pp. 35-44.

15. Rutledge, G.C., Shin, Y.M., Brenner, M.P., Hohman, M.M. Experimental Characterization of Electrospinning: the Electrically Forced Jet and Instabilities Polymer 42 2001: pp. 9955-9967.

16. Yao, L., Haas, T., Guiseppi-Elie, A., Bowlin, G.L., Simpson, D.G., Wnek, G.E. Electrospinning and Stabilization of Fully Hydrolyzed Poly(vinyl alcohol) Fibers Chemistry of Materials 15 2003: pp. 1860-1864. https://doi.org/10.1021/cm0210795

17. Zeng, J., Hou, H., Wendorff., J.H., Greiner, A. Electrospun Poly(vinyl alcohol)/Poly(acrylic acid) Fibers $\begin{array}{lll}\text { with Excellent Water-stability e-Polymers } 078 & \end{array}$ 2004: pp. $1-8$. 
18. Kumeta, K., Nagashima, I., Matsui, S., Mizoguchi, K. Crosslinking Reaction of Poly(vinyl alcohol) with Poly(acrylic acid) (PAA) by Heat Treatment: Effect of Neutralization of PAA Journal of Applied Polymer Science 90 2003: pp. 2420-2427. https://doi.org/10.1002/app.12910

19. Zeng, J., Hou, H., Wendorff, J.H., Greiner, A. PhotoInduced Solid-State Crosslinking of Electrospun Poly(vinyl alcohol) Fibers Macromolecular Rapid Communications 26 2005: pp. 1557-1562.

https://doi.org/10.1002/marc.200500545

20. Dror, Y., Salalha, W., $\quad$ Khalfin, R., Cohen, Y., Yarin, A.L., Zussman, E. Carbon Nanotubes Embedded in Oriented Polymer Nanofibers by Electrospinning Langmuir 19 2003: pp. 7012-7020.

21. Salalha, W., Dror, Y., Khalfin, R., Cohen, Y., Yarin, A.L., Zussman, E. Single-Walled Carbon Nanotubes Embedded in Oriented Polymeric Nanofibers by Electrospinning Langmuir 20 2004: pp. 9852-9855.

22. Lim, J., Yi, G., Moon, J., Heo, C., Yang, S. Fabrication of One-dimensional Colloidal Assemblies from Electrospun Nanofibers Langmuir 22 2006: pp. 3445-3449.

23. Liu, Y., Koops, G.H., Strathmann, H. Characterization of Morphology Controlled Polyethersulfone Hollow Fiber Membranes by the Addition of Polyethylene Glycol to the Dope and Bore Liquid Solution Journal of Membrane Science 223 2003: pp. 187-199.

24. Wang, Z., Xiao, C., Wu, C., Han, H. High-performance Polyethylene Glycol-coated Solid-phase Microextraction Fibers Using Sol-gel Technology Journal of Chromatography A 893 2000: pp. 157-168. https://doi.org/10.1016/S0021-9673(00)00692-0

25. Wongchitphimon, S., Wang, R., Jiraratananon, R., Shi, L., Loh, C.H. Effect of Polyethylene Glycol (PEG) as an Additive on the Fabrication of Polyvinylidene Fluorideco-hexafluropropylene (PVDF-HFP) Asymmetric Microporous Hollow Fiber Membranes Journal of Membrane Science 369 2011: pp. 329-338.

26. Zeugolis, D.I., Paul, R.G., Attenburrow, G. Extruded Collagen-Polyethylene Glycol Fibers for Tissue Engineering Applications Journal of Biomedical Materials Research 85B 2008: pp. 343-352.
27. Sungkaew, S., Thammakhet, C., Thavarungkul, P., Kanatharana, P. A New Polyethylene Glycol Fiber Prepared by Coating Porous Zinc Electrodeposited onto Silver for Solid-phase Microextraction of Styrene Analytica Chimica Acta 664 2010: pp. 49-55.

28. Chen, C., Wang, L., Huang, Y. Electrospinning of Thermos-regulating Ultrafine Fibers Based on Polyethylene Glycol/Cellulose Acetate Composite Polymer 48 2007: pp. $5202-5207$.

29. Fortunato, G., Guex, A.G., Popa, A.M., Rossi, R.M., Hufenus, R. Molecular Weight Driven Structure Formation of PEG Based E-spun Polymer Blend Fibres Polymer 55 2014: pp. 3139-3148.

30. Deitzel, J.M., Kleinmeyer, J., Harris, D., Beck Tan, N.C. The Effect of Processing Variables on the Morphology of Electrospun Nanofibers and Textiles Polymer 42 2001: pp. $261-272$.

31. Jaeger, R., Bergschoof, M., Martini, I., Batlle, C., Schönherr, H., Vancso, G.J. Electrospinning of Ultra-thin Polymer Fibers Macromolecular Symposium 1998: pp. $127141-127150$.

32. Peer, P., Filip, P, Polaskova, M., Kucharczyk, P., Pavlinek, V. The Influence of Sonication of Poly(ethylene oxide) Solutions to the Quality of Resulting Electrospun Nanofibrous Mats Polymer Degradation and Stability 126 2016: pp. $101-106$. https://doi.org/10.1016/j.polymdegradstab.2016.02.002

33. Malasauskiene, J., Milasius, R. Mathematical Analysis of the Diameter Distribution of Electrospun Nanofibers Fibers and Textiles in Eastern Europe 18 2010: pp. 45-48.

34. Malasauskiene, J., Milasius, R. Investigation and Estimation of Structure of Web from Electrospun Nanofibres Journal of Nanomaterialsk 2013: pp. 1-6.

35. Malasauskiene, J., Milasius, R., Kuchanauskaite, E. Possibilities for the Estimation of Electrospun Nanofibre Diameter Distribution by Normal (Gaussian) Distribution Fibres and Textiles in Eastern Europe 24 2016: pp. $23-28$. https://doi.org/10.5604/12303666.1191423

36. Cai, Y., Gevelber, M. Analysis of Bending Region Physics in Determining Electrospun Fiber Diameter: Effect of Relative Humidity on Evaporation and Force Balance Journal of Materials Science 52 2017: pp. 2605-2627. 